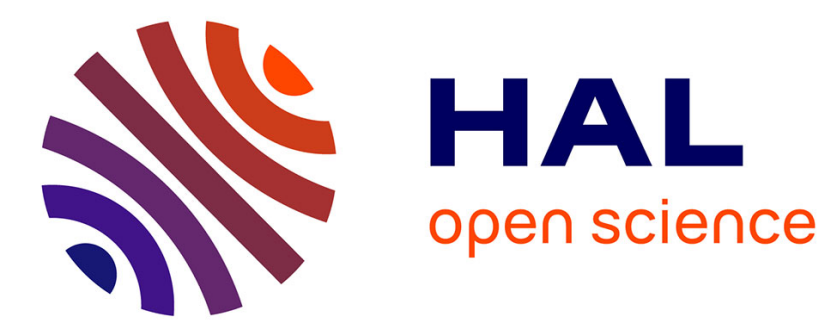

\title{
Analytic gradients for the combined sr-DFT/lr-MP2 method: Application to weakly bound systems
}

Sylvain Chabbal, Hermann Stoll, Hans-Joachim Werner, Thierry Leininger

\section{To cite this version:}

Sylvain Chabbal, Hermann Stoll, Hans-Joachim Werner, Thierry Leininger. Analytic gradients for the combined sr-DFT/lr-MP2 method: Application to weakly bound systems. Molecular Physics, 2010, 108 (24), pp.3373. 10.1080/00268976.2010.510807 . hal-00633605

\section{HAL Id: hal-00633605 https://hal.science/hal-00633605}

Submitted on 19 Oct 2011

HAL is a multi-disciplinary open access archive for the deposit and dissemination of scientific research documents, whether they are published or not. The documents may come from teaching and research institutions in France or abroad, or from public or private research centers.
L'archive ouverte pluridisciplinaire HAL, est destinée au dépôt et à la diffusion de documents scientifiques de niveau recherche, publiés ou non, émanant des établissements d'enseignement et de recherche français ou étrangers, des laboratoires publics ou privés. 


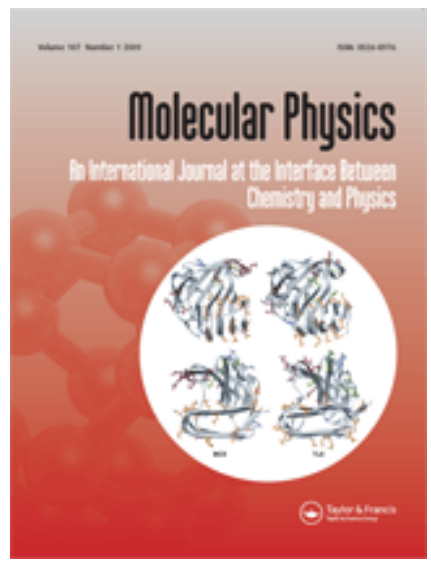

\section{Analytic gradients for the combined sr-DFT/Ir-MP2 method: Application to weakly bound systems}

\begin{tabular}{|r|l|}
\hline Journal: & Molecular Physics \\
\hline Manuscript ID: & TMPH-2010-0224 \\
\hline Manuscript Type: & Full Paper \\
\hline Author: & 07-Jun-2010 \\
\hline & $\begin{array}{l}\text { Complete List of Authors: } \\
\text { CHABBAL, Sylvain; Laboratoire de Chimie et Physique Quantiques, } \\
\text { Stoll, Hermann; Institute for Theoretical Chemistry } \\
\text { LEININGER, Thierry; Laboratoire de Chimie et Physique Quantiques, } \\
\text { Université Toulouse 3 }\end{array}$ \\
\hline Keywords: & $\begin{array}{l}\text { short-range/long-range separation, analytical gradients, DFT/MP2, } \\
\text { non-bonded complexes }\end{array}$ \\
\hline & \\
\hline $\begin{array}{l}\text { Note: The following files were submitted by the author for peer review, but cannot be converted } \\
\text { to PDF. You must view these files (e.g. movies) online. }\end{array}$ \\
\hline source files.zip \\
\hline
\end{tabular}

\section{SCHOLARONE \\ Manuscripts}


Molecular Physics

Vol. 00, No. 00, Month 200x, 1-13

\title{
RESEARCH ARTICLE
}

\section{Analytic gradients for the combined $s r$-DFT/lr-MP2 method: Application to weakly bound systems}

\author{
Sylvain CHABBAL ${ }^{a}$, Hermann STOLL ${ }^{b}$, Hans-Joachim WERNER ${ }^{b}$ and Thierry \\ LEININGER $^{a *}$ \\ ${ }^{a}$ Laboratoire de Chimie et Physique Quantiques, Université de Toulouse, CNRS, \\ Toulouse, France; ${ }^{b}$ Institut für Theoretische Chemie, Universität Stuttgart, Stuttgart, \\ Germany \\ (4 June 2010)
}

\begin{abstract}
The formalism for analytical gradients in short-range density functional schemes with longrange ab-initio corrections $(s r$-DFT/lr-ab initio) is presented. On the density-functional side, both $s r$-LDA and $s r$-PBE are available, while on the ab-initio side $l r-\mathrm{HF}$ and $l r-\mathrm{MP} 2$ are possible. Details of the implementation in the Molpro package are given. Results from test calculations using different basis sets on weakly bound systems of the HB6/04, DI6/04, CT7/04 and WI9/04 databases are presented.
\end{abstract}

\section{Keywords}

short-range/long-range separation; analytical gradients; DFT/MP2; non-bonded complexes

\section{Introduction}

For theoretical calculations aiming at the treatment of medium to large systems, energy gradients are indispensable as a tool for geometry optimization. The determination of analytical gradients has been pioneered in quantum chemistry by Pulay [1], and gradients are available nowadays in practically all density-functional and ab-initio wavefunction-based program packages, see [2] for a review.

It is clear that the emergence of new methods should be accompanied by efforts to also code corresponding energy gradients. The present paper is devoted to the development and implementation of a gradient code for the class of recently developed range-separated hybrid methods which feature a coupling between a density-functional (DFT) treatment for short inter-electronic distances $r_{i j}$ and a wavefunction-based one for large $r_{i j}$, see e.g.[3-5]. The introduction of such a coupling should help to improve the accuracy of standard DFT which is not reliable for the treatment of long-range correlation effects; it should also help to reduce the heavy computational effort of wavefunction-based post-Hartree-Fock (post-HF) methods which is due to difficulties in describing the inter-electronic cusp of the wavefunction.

\footnotetext{
${ }^{*}$ Corresponding author. Email: Thierry.Leininger@irsamc.ups-tlse.fr
}

ISSN: 0040-5167 print/ISSN 1754-2278 online

(C) 200x Taylor \& Francis

DOI: $10.1080 / 0040516$ YYxxxxxxxx

http://www.informaworld.com 
When calculating gradients for a hybrid scheme like $s r$-DFT/lr-MP2 (short-range DFT coupled with long-range Møller-Plesset second-order perturbation theory), it seems natural to make use of the already available machinery for standard DFT and MP2 gradients. However, the coupling leads to some new features to be discussed in the present paper. The paper is organized as follows: Section 2 contains summaries of the range-separated hybrid method and of the MP2 gradient formulae, and then describes the changes to be introduced in the latter ones for an account of short-range DFT. Application of the newly implemented gradient code for geometry optimization at the $s r$-DFT/lr-MP2 level for a benchmark set of weakly bound systems [6] is discussed in Section 3, followed by an outlook in Section 4.

\section{Theory}

\subsection{Hybrid method}

Here we will briefly review the basic equations leading to the electronic energy expression in the range-separated hybrid formalism. The range-separated electron interaction is obtained by splitting the interelectronic potential in two terms [3]

$$
V_{e e}=\sum_{i<j} \frac{\operatorname{erfc}\left(\mu r_{i j}\right)}{r_{i j}}+\sum_{i<j} \frac{\operatorname{erf}\left(\mu r_{i j}\right)}{r_{i j}}=V_{e e}^{S R}+V_{e e}^{L R}
$$

where erf is the standard error function and erfc its complement. The relative weights of the short-range and long-range parts are controlled by the $\mu\left(\mathrm{bohr}^{-1}\right)$ parameter which, in a sense, may be considered as an inverse splitting "distance" for the electronic correlation treatment. For $\mu=0$, the pure Kohn-Sham result is recovered and for $\mu \rightarrow \infty$, the ab-initio limit.

In the Levy constrained-search formalism [7], the ground-state energy reads

$$
E_{0}=\min _{\rho}\left(\min _{\Psi \rightarrow \rho}\left\langle\Psi\left|T+V_{n e}+V_{e e}\right| \Psi\right\rangle\right)
$$

with $T$ the kinetic energy operator, $V_{n e}$ the electron-nuclear potential, and $\rho$ the electron density. By replacing the interelectronic potential with equation (1), we have

$$
E_{0}=\min _{\rho}\left(E_{0}^{S R}[\rho]+\min _{\Psi \rightarrow \rho}\left\langle\Psi\left|T+V_{n e}+V_{e e}^{L R}\right| \Psi\right\rangle\right),
$$

where $E_{0}^{S R}[\rho]$ is a universal short-range energy functional which is defined as

$$
E_{0}^{S R}[\rho]=\min _{\Psi \rightarrow \rho}\left\langle\Psi\left|T+V_{e e}\right| \Psi\right\rangle-\min _{\Psi \rightarrow \rho}\left\langle\Psi\left|T+V_{e e}^{L R}\right| \Psi\right\rangle,
$$

and can be rewritten as

$$
E_{0}^{S R}[\rho]=J^{S R}[\rho]+E_{X C}^{S R}[\rho]
$$

Here $J^{S R}$ is the short-range Coulomb energy expressed as

$$
J^{S R}[\rho]=\frac{1}{2} \int \rho\left(r_{1}\right) \frac{\operatorname{erfc}\left(\mu r_{12}\right)}{r_{12}} \rho\left(r_{2}\right) d r_{1} d r_{2}
$$


and $E_{X C}^{S R}$ is the exchange-correlation term which in the local-density approximation (LDA) reads

$$
E_{X C}^{S R}[\rho]=\int \rho \epsilon_{X C}^{S R}(\rho) d r
$$

The short-range exchange-correlation energy per particle, $\epsilon_{X C}^{S R}$, can be obtained from the difference of the exchange-correlation energies per particle of the electron gas with full and long-range interelectronic interaction, respectively. Generalized gradient approximations (GGA) for the short-range functional are also available [5].

If $\Psi^{L R}$ is the wavefunction obtained from the double minimization in equation (3), the ground-state energy reads

$$
E_{0}=E_{0}^{S R}\left[\rho_{\Psi^{L R}}\right]+\left\langle\Psi^{L R}\left|T+V_{n e}+V_{e e}^{L R}\right| \Psi^{L R}\right\rangle
$$

where $\rho_{\Psi^{L R}}$ is the density associated with $\Psi^{L R}$. If we assume the form of a single Slater determinant for $\Psi^{L R}$, its orbitals are eigenfunctions of a Fock-like operator $F$ which contains the functional derivative of $E_{0}^{S R}[\rho], \delta E_{0}^{S R} / \delta \rho$, as well as Coulomb and exchange operators formed with $V_{e e}^{L R}$, in addition to the usual one-electron operators. We can then improve on $\Psi^{L R}$, at the MP2 level, by using $H_{0}=\sum_{i} F(i)$ as zeroth-order Hamiltonian and $H_{1}=T+V_{n e}+V_{e e}^{L R}+\delta E_{0}^{S R} / \delta \rho-H_{0}$ as perturbation. The energy gradients relative to the atomic coordinates $X=$ $\left(x_{1}, y_{1}, z_{1} ; x_{2}, y_{2}, z_{2} ; \ldots\right)$ are obtained by differentiation of the energy functional with respect to $X$. The energy depends on the mono- and bi-electronic integrals in the AO basis, the MO coefficients, and the MP2 amplitudes. (The short-range functional also depends on the $\mathrm{AO}$ basis and the MO coefficients via the density $\rho$.) Thus, the evaluation of $\frac{\partial E_{0}}{\partial X}$ requires partial derivatives with respect to all these quantities (with the exception of the MP2 amplitudes if the MP2 Hylleraas functional is variationally optimized).

The gradients for the hybrid $s r$-DFT/lr-MP2 case are obtained by combining (and modifying) the standard DFT and MP2 contributions. This is straightforward for the DFT part. For the MP2 part, the code has to be modified by using longrange bi-electronic integrals and introducing short-range contributions to the Fock matrix. In the next section, we give the resulting equations and details concerning the implementation in the Molpro [8] code.

\subsection{MP2 gradients}

The following notation for the orbital indices is used throughout the text: $\mu, \nu, \ldots$ refers to atomic orbitals (AO), $i, j, \ldots$ to occupied molecular orbitals (MO), $a, b, \ldots$ to virtual unoccupied orbitals and $p, q, r, s, \ldots$ to any molecular orbital. The MOs $\phi$ are expressed as linear combinations of atomic orbitals $\chi, i e, \phi_{i}=\sum_{\mu} C_{\mu i} \chi_{\mu}$. We will also use the following conventions : ${ }^{x}$ denotes the derivative with respect to the atomic coordinates; a superscript in parenthesis, ${ }^{(x)}$, denotes the corresponding quantity evaluated with integral derivatives but using the initial SCF coefficients and MP2 amplitudes, while a superscript in square bracket ${ }^{[x]}$ denotes a quantity evaluated with derivatives of SCF coefficient matrices, but using the zeroth-order integrals. Below, we briefly review the basic equations for the MP2 gradients which have to be modified in the $s r$-DFT/lr-MP2 case. 
The derivative of the MP2 energy with respect to atomic coordinates is $[9,10]$ :

$$
E_{H F}^{x}+E_{M P 2}^{x}=\sum_{\mu \nu} d_{\mu \nu} h_{\mu \nu}^{(x)}+\frac{1}{2} \sum_{\mu \nu \rho \sigma} D_{\mu \nu, \rho \sigma}(\mu \nu \mid \rho \sigma)^{(x)}+\sum_{\mu \nu} X_{\mu \nu} S_{\mu \nu}^{(x)}
$$

with $h^{(x)}$ and $(\mu \nu \mid \rho \sigma)^{(x)}$ the one- and two-electron integral derivatives, $S^{(x)}$ the overlap matrix derivative, and $d, D$ the effective first- and second-order density matrices, respectively. The effective one-electron density matrix $d$ is given by:

$$
d_{\mu \nu}=d_{\mu \nu}^{(0)}+d_{\mu \nu}^{(2)}+\sum_{p q} C_{\mu p} z_{p q} C_{\mu q}
$$

where $d^{(0)}$ is the SCF density matrix, $d^{(2)}$ the MP2 correction to it, and $z$ is the Lagrangian multiplier for zeroing out Fock matrix elements between occupied and virtual orbitals. The second-order density matrix is

$$
D_{\mu \nu, \rho \sigma}=4 \sum_{i j} C_{\mu i} \bar{T}_{\nu \sigma}^{i j} C_{\rho j}+2\left(d_{\mu \nu}-\frac{1}{2} d_{\mu \nu}^{(0)}\right) d_{\rho \sigma}^{(0)}-\left(d_{\mu \rho}-\frac{1}{2} d_{\mu \rho}^{(0)}\right) d_{\nu \sigma}^{(0)}
$$

where $T$ is the MP2 amplitude matrix and $\bar{T}$ the contravariant amplitude matrix defined as $\bar{T}_{i j}=2 T_{i j}-T_{j i}$. Finally, $X$ is defined as

$$
X_{\mu \nu}=-2 \sum_{i j} C_{\mu i} F_{i j} C_{\nu j}-\frac{1}{2} \sum_{r s} C_{\mu r} x_{r s} C_{\nu s}
$$

where $F$ is the Fock matrix and $x$ is the Lagrangian multiplier for keeping orbital orthogonality. The Lagrangian multipliers $x$ and $z$ are determined from the condition that the MP2 Lagrangian is stationary with respect to orbital changes. In the case of $z$ this leads to the well-known coupled-perturbed Hartree-Fock (CPHF) [11-13] equations which have to be solved iteratively. Both the CPHF equations and the equations for $x$ depend linearly on derivatives of the Fock operator with respect to the matrix $U-1$ where $U$ is a unitary matrix of orbital transformations.

\subsection{Adaptation to the hybrid case}

Our implementation is based on the existing code for the DFT and MP2 gradients in Molpro [8]. However, the calculation of the analytical gradients in the hybrid sr$\mathrm{DFT} / l r$-MP2 scheme requires several modifications which can be illustrated with the formulae of the preceding paragraphs. A summary of these changes is given below:

(1) As a first step, all the bi-electronic integrals in the MP2 gradient of equation (9) have to be replaced with corresponding ones involving $V_{e e}^{L R}$ instead of $V_{e e}$. Also, the pre-screening of the bi-electronic integral derivatives has to be changed.

(2) Derivatives of the short-range Coulomb energy, $J^{S R(x)}$, and short-range exchange-correlation energy, $E_{X C}^{S R(x)}$, must be added to the long-range MP2 gradient.

(3) In the Fock operator $F$ appearing in $X$ and the equations determining the Lagrangian multipliers $x$ and $z$, its short-range part, $F^{S R}$, must be taken into account. 
(4) In the CPHF equations (and the equations for the Lagrangian multiplier $x$ ) also orbital derivatives of $F^{S R[x]}$ are needed.

\subsubsection{Modification of the pre-screening}

The computation of the atomic integral derivatives in Molpro relies on the Alaska program [14] which uses a pre-screening for two-electron evaluation. In the case of long-range bi-electronic integrals, this pre-screening has to be modified to introduce the sr/lr coupling parameter $\mu$. Thus, equation (8) in the original paper of Lindh [14] should be replaced by the following expression :

$$
P_{\zeta \eta, a b c d}^{\prime}=2 \pi^{\frac{5}{2}} \kappa_{a b} \kappa_{c d} \frac{1}{\sqrt{\eta+\zeta+\left(\frac{\zeta+\eta}{\mu^{2}}\right)}} \times P_{\eta \zeta, a b c d}
$$

where $\zeta, \eta, \kappa_{a b}, \kappa_{c d}$ and $P_{\eta \zeta, a b c d}$ are quantities depending on the Gaussian basis functions (more details can be found in [15]). Of course for the $\mu \rightarrow \infty$ limit, the standard prescreening is recovered.

\subsubsection{Addition of the $J^{S R(x)}$ and $E_{X C}^{S R(x)}$ contributions}

The derivative of the short-range Coulomb energy, for fixed density matrices, has to be added to the long-range MP2 gradient. This term can be written as (cf. equation (9) and second term in equation (11)):

$$
J^{S R(x)}=\sum_{\mu \nu \rho \sigma}\left(d_{\mu \nu}-\frac{1}{2} d_{\mu \nu}^{(0)}\right) d_{\rho \sigma}^{(0)}(\mu \nu \mid \rho \sigma)_{S R}^{(x)}
$$

where $(\mu \nu \mid \rho \sigma)_{S R}^{(x)}$ are the short-range two-electron integral derivatives, obtained from the difference between full-range and long-range integrals. Of course, this adds to the corresponding term of the long-range MP2 gradient to yield the gradient of the Coulomb energy with full interelectronic interaction.

With regard to short-range exchange-correlation, we first need the Hartree-Fock contribution for fixed SCF density matrix $d^{(0)}$. As other DFT contributions, it is calculated by summation over grid points $\lambda$ with weights $\omega_{\lambda}$, and reads for the LDA case as follows:

$$
\begin{aligned}
E_{X C}^{S R}\left[\rho^{(0)}\right]^{(x)} & =\sum_{\lambda} \omega_{\lambda}^{(x)} \rho_{\lambda}^{(0)} \epsilon_{X C}^{S R}\left(\rho_{\lambda}^{(0)}\right)+\sum_{\lambda} \omega_{\lambda} V_{X C}^{S R}\left(\rho_{\lambda}^{(0)}\right)\left(\rho_{\lambda}^{(0)}\right)^{(x)} \\
& =\sum_{\lambda} \omega_{\lambda}^{(x)} \rho_{\lambda}^{(0)} \epsilon_{X C}^{S R}\left(\rho_{\lambda}^{(0)}\right)+\sum_{\mu \nu} d_{\mu \nu}^{(0)} \sum_{\lambda} \omega_{\lambda} V_{X C}^{S R}\left(\rho_{\lambda}^{(0)}\right)\left(\chi_{\mu}^{\lambda} \chi_{\nu}^{\lambda}\right)^{(x)}
\end{aligned}
$$

where $V_{X C}^{S R}=d\left(\rho \epsilon_{X C}^{S R}(\rho)\right) / d \rho$ is the short-range exchange-correlation potential; $\omega_{\lambda}^{(x)},\left(\rho_{\lambda}^{(0)}\right)^{(x)}$ describe the changes due to the change of the grid points and the values of the basis functions at the grid points, respectively; $\chi_{\mu}^{\lambda}$ is the value of basis function $\chi_{\mu}$ at grid point $\lambda$.

We then have a second contribution which originates from the MP2 Hylleraas functional and is related to the Fock operator (cf. equation (9) and third term in 
equation (11)):

$$
\begin{aligned}
\sum_{\mu \nu} \tilde{d}_{\mu \nu}\left\langle\mu\left|V_{X C}^{S R}\left(\rho^{(0)}\right)\right| \nu\right\rangle^{(x)} & =\sum_{\lambda} \omega_{\lambda}^{(x)} V_{X C}^{S R}\left(\rho_{\lambda}^{(0)}\right) \tilde{\rho}_{\lambda} \\
& +\sum_{\lambda} \omega_{\lambda}\left(V_{X C}^{S R^{\prime}}\left(\rho_{\lambda}^{(0)}\right)\left(\rho_{\lambda}^{(0)}\right)^{(x)} \tilde{\rho}_{\lambda}+V_{X C}^{S R}\left(\rho_{\lambda}^{(0)}\right) \tilde{\rho}_{\lambda}^{(x)}\right)(16)
\end{aligned}
$$

Here, $\tilde{d}=d-d^{(0)}, \tilde{\rho}=\sum_{\mu \nu} \tilde{d}_{\mu \nu} \chi_{\mu} \chi_{\nu}$, and $V_{X C}^{S R^{\prime}}(\rho)=d V_{X C}^{S R} / d \rho$. The evaluation of $\left(\rho_{\lambda}^{(0)}\right)^{(x)}$ and $\tilde{\rho}_{\lambda}^{(x)}$ proceeds as in equation (15).

\subsubsection{Expression of the hybrid Fock operator}

Using the standard MP2 code with bi-electronic integrals formed over $V^{L R}$ leads to a Fock operator where short-range contributions are missing. Adding the contributions from the short-range Coulomb and exchange-correlation potentials leads to a sum of pure ab-initio $\left(F^{L R}\right)$ and DFT $\left(F^{S R}\right)$ contributions :

$$
F=F^{L R}+F^{S R}
$$

with

$$
\begin{aligned}
& F_{\mu \nu}^{L R}=h_{\mu \nu}+\sum_{\rho \sigma} d_{\mu \nu}\left((\mu \nu \mid \rho \sigma)_{L R}-\frac{1}{2}(\mu \rho \mid \nu \sigma)_{L R}\right) \\
& F_{\mu \nu}^{S R}=\sum_{\rho \sigma} d_{\mu \nu}(\mu \nu \mid \rho \sigma)_{S R}+\sum_{\lambda} \omega_{\lambda} V_{X C}^{S R}\left(\rho^{\lambda}\right) d_{\mu \nu} \chi_{\mu}^{\lambda} \chi_{\nu}^{\lambda}
\end{aligned}
$$

where the $\chi_{\mu}^{\lambda}$ are basis function values at grid points. In all expressions involving $F$ in the MP2 gradient expression, the $F^{S R}$ have to be supplemented to the $F^{L R}$, with density matrices $d^{(0)}$ taken from the preceeding $s r$-DFT/lr-HF calculation.

\subsubsection{CPHF equations}

As already mentioned above, the determination of the Lagrangian multipliers which add to the MP2 Hylleraas functional requires the calculation of the derivative, with respect to orbital changes, of an expression of the form $\operatorname{tr}(F \tilde{d})^{[x]}$ where $F$ is the Fock matrix and $\tilde{d}=d-d^{(0)}$. We are concerned here with the extra terms which arise due to the orbital dependence of $F$.

The extra term from the short-range Coulomb part in $F^{S R}$ is completely analogous to that in $F^{L R}$ and can simply be handled by using integrals with full interelectronic interaction. For the short-range exchange-correlation potential we have

$$
\begin{aligned}
\operatorname{tr}\left(V_{X C}^{S R[x]} \tilde{d}\right) & =\sum_{\mu \nu} \tilde{d}_{\mu \nu}<\mu\left|V_{X C}^{S R}\left(\rho^{(0)}\right)\right| \nu>^{[x]} \\
& =\sum_{\lambda} \omega_{\lambda} \tilde{\rho}_{\lambda} V_{X C}^{S R^{\prime}}\left(\rho_{\lambda}^{(0)}\right)\left(\rho_{\lambda}^{(0)}\right)^{[x]} \\
& =\sum_{\mu \nu}\left(d_{\mu \nu}^{(0)}\right)^{[x]} \sum_{\lambda} \omega_{\lambda} \tilde{\rho}_{\lambda} V_{X C}^{S R^{\prime}}\left(\rho_{\lambda}^{(0)}\right) \chi_{\mu}^{\lambda} \chi_{\nu}^{\lambda}
\end{aligned}
$$

where $\tilde{\rho}=\sum_{\mu \nu} \tilde{d}_{\mu \nu} \chi_{\mu} \chi_{\nu}$ (as above), and the $\chi_{\mu}^{\lambda}$ are the values of the basis functions at the grid points $\lambda$. 
The short-range terms discussed above appear linearly in the equation for $x$ and also in the equations for the CPHF iterations. The CPHF part is the most timeconsuming step of our implementation. Indeed, all the density dependent quantities are recomputed on the DFT grid at each CPHF iteration, and the added modifications scale as $n_{\text {grid-points }} \times N^{2}$, where $N$ is the number of basis functions, $n_{\text {grid-points }}$ the number of grid points.

All these modifications have been implemented and are available in the 2009.2 development version of the Molpro package. The code has been checked against numerical calculations (using a 4-point formula), for several molecular test systems using different basis sets. The following functionals are available : ecerf [16-19] and exerf [20], which are based on the LDA approximation, and ecerfpbe and exerfpbe [21] wich are based on the GGA/PBE approximation.

In all cases, the difference between numerical and analytical gradient values were less than $1.10^{-7}$ au.

It is worthwhile noting that as a byproduct of this code it is also possible to perform geometry optimization at the $s r$-DFT/lr-HF level. The implementation has been done in such a way that the present method can also be applied in the framework of local-correlation formalism [9].

\section{Application to weakly bound systems}

In this section, we present results obtained for some of the benchmark databases of Zhao and Truhlar [6]. They comprise a hydrogen bonding database (HB6/04), which consists of six dimers: $\left(\mathrm{NH}_{3}\right)_{2},(\mathrm{HF})_{2},\left(\mathrm{H}_{2} \mathrm{O}\right)_{2}$, $\mathrm{NH}_{3} \cdots \mathrm{H}_{2} \mathrm{O}$, $\left(\mathrm{HCONH}_{2}\right)_{2}$, and $(\mathrm{HCOOH})_{2}$. The second one is a charge transfer database $(\mathrm{CT} 7 / 04)$, made of the seven systems: $\mathrm{C}_{2} \mathrm{H}_{4} \cdots \mathrm{F}_{2}, \mathrm{NH}_{3} \cdots \mathrm{F}_{2}$, $\mathrm{C}_{2} \mathrm{H}_{2} \cdots \mathrm{ClF}, \mathrm{HCN} \cdots \mathrm{ClF}, \mathrm{NH}_{3} \cdots \mathrm{Cl}_{2}, \mathrm{H}_{2} \mathrm{O} \cdots \mathrm{ClF}$, and $\mathrm{NH}_{3} \cdots \mathrm{ClF}$. The third one considered is a dipole interaction database (DI6/04), with 6 molecules: $\left(\mathrm{H}_{2} \mathrm{~S}_{2},(\mathrm{HCl})_{2}, \mathrm{H}_{2} \mathrm{~S} \cdots \mathrm{HCl}, \mathrm{CH}_{3} \mathrm{Cl} \cdots \mathrm{HCl}, \mathrm{CH}_{3} \mathrm{SH} \cdots \mathrm{HCN}, \mathrm{CH}_{3} \mathrm{SH} \cdots \mathrm{HCl}\right.$. The last one is a weak interaction database (WI9/04) consisting of nine compounds: $\mathrm{HeNe}, \mathrm{HeAr}, \mathrm{Ne}_{2}, \mathrm{NeAr}, \mathrm{CH}_{4} \cdots \mathrm{Ne}, \mathrm{C}_{6} \mathrm{H}_{6} \cdots \mathrm{Ne},\left(\mathrm{CH}_{4}\right)_{2},\left(\mathrm{C}_{2} \mathrm{H}_{2}\right)_{2}$ and $\left(\mathrm{C}_{2} \mathrm{H}_{4}\right)_{2}$. We chose these databases because they were already used as tests systems for hybrid methods without geometry optimisation by Goll et al. [4]. Three different basis-sets were used for our calculations: DIDZ [22, 23] (an abreviation for 6-31+G(d,p)), MG3S [24-29] (approximately corresponding to 6-311+G(3df,2p)), and aug-cc-pVTZ [30-32]. We compared values from different hybrid calculations to the W1 references obtained by Zhao and Truhlar [6] and by Boese and Martin [33].

All geometry optimisations with the $s r$-DFT/lr-MP2 hybrid gradients were done starting from the reference geometries (optimized with a MC-QCISD method) available on the Truhlar group website [34]. For all of the above molecular systems, the dissociation energies $D_{e}$ have then been computed for the optimized geometries using the $s r$-LDA/lr-MP2 and $s r$-PBE/lr-MP2 hybrid methods. Results for optimized geometries and dissociation energies are available with and without counterpoise correction. In each case the mean absolute deviation (MAD) with respect to the reference values has been calculated.

Typical values of the $\mu$ parameter (equation (1)) used in the literature lie in the range of between 0.3 and $0.5 \mathrm{bohr}^{-1}$. (Figure 1) shows the evolution of the dissociation energy for the $\mathrm{H}_{2} \mathrm{O} \cdots \mathrm{ClF}$ system in the $s r$-LDA/lr-MP2 case with the hybrid parameter $\mu$. It can be seen that the convergence to the MP2 value is 


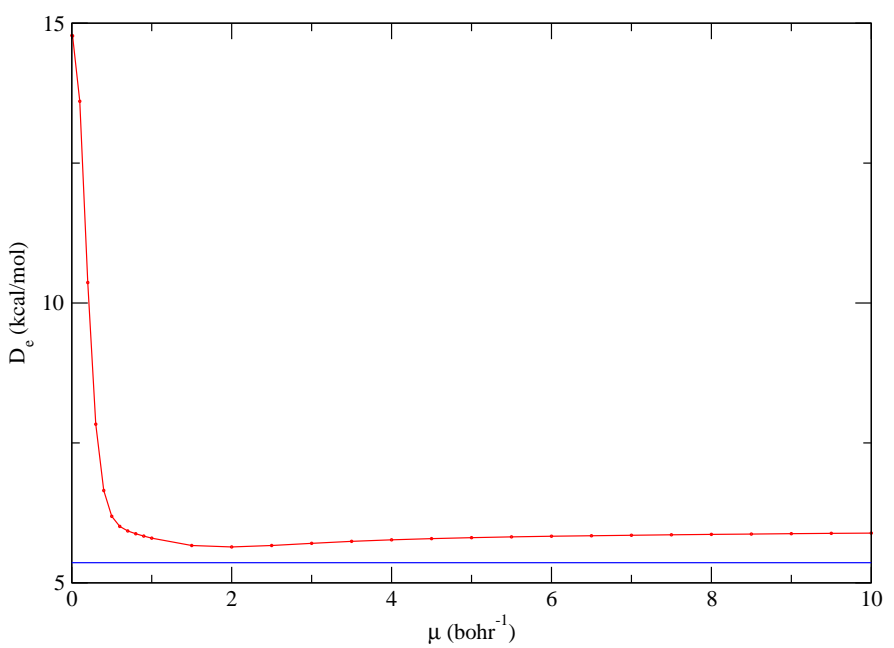

Figure 1. Evolution of $D_{e}(\mathrm{kcal} / \mathrm{mol})$ with $\mu$ for the $H_{2} O \cdots C l F$ complex. Results refer to LDA/MP2 calculations with $6-311+\mathrm{G}(3 \mathrm{df}, 2 \mathrm{p})$ basis sets.

already achieved for $\mu=0.5 \mathrm{bohr}^{-1}$ (the deviation is less than $5 \%$ ). This type of convergence has been observed for all the calculations presented here. Thus, all the results below are given for this value of $\mu$.

\section{1. $C T 7 / 04$.}

The results for this database are summarized in (Table 1). Using the counterpoise correction yields results closer to the reference values. Indeed, the LDA/MP2 MAD starting from $0.63(\mathrm{kcal} / \mathrm{mol})$ without $\mathrm{CP}$ is reduced to $0.43(\mathrm{kcal} / \mathrm{mol})$. The same trend is observed for the other methods. Therefore, we used the counterpoise correction for all following discussion.

For these compounds, the MAD obtained with LDA $/ \mathrm{MP} 2(0.43 \mathrm{kcal} / \mathrm{mol})$ is not very different from the MP2 one $(0.26 \mathrm{kcal} / \mathrm{mol})$, ie, our results with the hybrid method are in good agreement with the reference values. As noted in Ref. [4], the slightly better performance of MP2 is to some extent due to an error cancellation between basis-set effects and errors of the method, while the LDA/MP2 values are already close to the basis-set limit (see below). The PBE/MP2 MAD (0.54 $\mathrm{kcal} / \mathrm{mol}$ ) is not quite as good as the LDA/MP2 one, but is still close to the MP2 one. It is important to notice the small difference between the MADs of the two hybrid methods. This means that we obtain nearly the same level of accuracy with different functionals as already pointed out by Goll et al. [4]. This is clearly not the case for standard DFT calculations. For instance, the MAD is $11.28(\mathrm{kcal} / \mathrm{mol})$ for LDA and $4.97(\mathrm{kcal} / \mathrm{mol})$ for PBE. We can also compare our hybrid results obtained after geometry optimisation with those of Ref. [4]. In the latter reference, the MAD for $\mathrm{PBE} / \mathrm{MP} 2$ without geometry optimisation is $0.66(\mathrm{kcal} / \mathrm{mol})$. The geometry optimisation then improves the results. Indeed, our value is $0.54(\mathrm{kcal} / \mathrm{mol})$. If one compares with the results obtained by Zhao et al. [6], it can be noticed that in the case of pure density functional calculations, geometry optimisation basically doubles the MAD. This is obviously not the case for hybrid geometry optimisation. 
Table 1. Dissociation energies (kcal/mol) for the CT7/04 compounds for different approaches using MG3S basis sets with (cp) and without (no-cp) counterpoise correction

\begin{tabular}{|c|c|c|c|c|c|c|c|c|c|c|c|}
\hline & \multicolumn{2}{|c|}{ LDA } & \multicolumn{2}{|c|}{ PBE } & \multicolumn{2}{|c|}{ LDA/MP2 } & \multicolumn{2}{|c|}{ PBE/MP2 } & \multicolumn{2}{|c|}{ MP2 } & \multirow[t]{2}{*}{ Ref $[6]$} \\
\hline & no-cp & $\mathrm{cp}$ & no-cp & $\mathrm{cp}$ & no-cp & $\mathrm{cp}$ & no-cp & $\mathrm{cp}$ & no-cp & $\mathrm{cp}$ & \\
\hline $\mathrm{C}_{2} \mathrm{H}_{4} \ldots \bar{F}_{2}$ & 17.25 & 10.32 & 4.92 & 4.43 & 1.16 & 0.77 & 1.25 & 0.87 & 1.56 & 0.86 & 1.06 \\
\hline$N H_{3} \ldots F_{2}$ & 20.22 & 19.50 & 14.3 & 13.76 & 1.71 & 1.28 & 1.87 & 1.46 & 1.99 & 1.33 & 1.81 \\
\hline $\mathrm{C}_{2} \mathrm{H}_{2} \ldots \mathrm{ClF}$ & 14.67 & 14.21 & 7.86 & 7.55 & 4.36 & 4.00 & 4.71 & 4.37 & 4.89 & 3.94 & 3.81 \\
\hline$H C N \ldots C l F$ & 14.21 & 13.55 & 6.90 & 6.52 & 5.81 & 5.45 & 5.97 & 5.65 & 5.72 & 4.87 & 4.86 \\
\hline $\mathrm{NH}_{3} \ldots \mathrm{Cl}_{2}$ & 15.51 & 15.05 & 9.62 & 9.22 & 5.19 & 4.72 & 5.51 & 5.06 & 5.59 & 4.65 & 4.88 \\
\hline $\mathrm{H}_{2} \mathrm{O} \ldots \mathrm{ClF}$ & 14.68 & 13.95 & 8.78 & 8.18 & 6.29 & 4. 80 & 6.49 & 5.88 & 6.00 & 4.80 & 5.36 \\
\hline $\mathrm{NH}_{3} \ldots \mathrm{ClF}$ & 25.45 & 24.80 & 17.98 & 17.52 & 12.10 & 11.34 & 12.49 & 11.82 & 11.95 & 10.38 & 10.62 \\
\hline MAD & 12.80 & 11.28 & 5.42 & 4.97 & 0.63 & 0.43 & 0.84 & 0.54 & 0.76 & 0.26 & \\
\hline
\end{tabular}

This fact is confirmed by (Table 2), which shows the evolution of the binding distance with geometry optimisation. Differences between the reference geometry and the optimized one obtained with hybrid methods are very small, contrary to pure LDA or PBE results. The MADs for hybrid methods are basically the same with and without counterpoise correction. This is not the case for pure DFT and MP2 methods.

Table 2. Intermolecular distances (A) for CT7/04 compounds with different approaches using MG3S basis sets with (cp) and without (no-cp) counterpoise correction.

\begin{tabular}{|c|c|c|c|c|c|c|c|c|c|c|c|}
\hline & \multicolumn{2}{|c|}{$\overline{\mathrm{LDA}}$} & \multicolumn{2}{|c|}{$\overline{\mathrm{PBE}}$} & \multicolumn{2}{|c|}{ LDA/MP2 } & \multicolumn{2}{|c|}{ PBE/MP2 } & \multicolumn{2}{|c|}{ MP2 } & \multirow[t]{2}{*}{ Ref [34] } \\
\hline & no-cp & $\mathrm{cp}$ & no-cp & $\mathrm{cp}$ & no-cp & $\mathrm{cp}$ & no-cp & $\mathrm{cp}$ & no-cp & $\mathrm{cp}$ & \\
\hline $\mathrm{C}_{2} \mathrm{H}_{4} \ldots \bar{F}_{2}$ & 2.272 & 2.287 & 2.490 & 2.510 & 3.014 & 3.095 & 2.960 & 3.054 & 2.912 & 3.020 & 3.053 \\
\hline $\mathrm{NH}_{3} \ldots \mathrm{F}_{2}$ & 1.859 & 1.864 & 1.932 & 1.937 & 2.678 & 2.730 & 2.629 & 2.673 & 2.646 & 2.743 & 2.696 \\
\hline$C_{2} H_{2} \ldots C l F$ & 2.371 & 2.381 & 2.529 & 2.542 & 2.827 & 2.846 & 2.790 & 2.806 & 2.800 & 2.865 & 2.876 \\
\hline$H C N \ldots C l F$ & 2.076 & 2.095 & 2.308 & 2.334 & 2.532 & 2.554 & 2.515 & 2.535 & 2.548 & 2.606 & 2.609 \\
\hline $\mathrm{NH}_{3} \ldots \mathrm{Cl}_{2}$ & 2.277 & 2.283 & 2.388 & 2.394 & 2.644 & 2.663 & 2.611 & 2.624 & 2.603 & 2.660 & 2.688 \\
\hline $\mathrm{H}_{2} \mathrm{O} \ldots \mathrm{ClF}$ & 2.246 & 2.255 & 2.369 & 2.380 & 2.476 & 2.494 & 2.461 & 2.483 & 2.512 & 2.564 & 2.557 \\
\hline $\mathrm{NH}_{3} \ldots \mathrm{ClF}$ & 2.120 & 2.127 & 2.195 & 2.204 & 2.2410 & 2.259 & 2.237 & 2.253 & 2.234 & 2.276 & 2.302 \\
\hline MAD & 0.509 & 0.498 & 0.367 & 0.354 & 0.053 & 0.042 & 0.082 & 0.051 & 0.075 & 0.022 & \\
\hline
\end{tabular}

In (Table 3), we compare the influence of different basis sets on $D_{e}$. One can notice a small variation $(\sim 0.2 \mathrm{kcal} / \mathrm{mol})$ of the MAD between the different basis sets. The most important binding energy variation between the basis sets (ie, between MG3S and DIDZ results) is of the same order of magnitude as that observed between DFT/MP2 and MP2 for the same basis set [4].

\begin{tabular}{|c|c|c|c|c|c|c|}
\hline \multirow[t]{2}{*}{$\mathrm{PBE} / \mathrm{MP} 2$} & \multicolumn{2}{|c|}{ DIDZ } & \multicolumn{2}{|c|}{ MG3S } & \multicolumn{2}{|c|}{ aug-cc-pVTZ } \\
\hline & no-cp & $\mathrm{cp}$ & no-cp & $\mathrm{cp}$ & no-cp & $\mathrm{cp}$ \\
\hline $\mathrm{C}_{2} \mathrm{H}_{4} \ldots F_{2}$ & 1.39 & 0.66 & 1.25 & 0.87 & 1.17 & 1.08 \\
\hline $\mathrm{NH}_{3} \ldots \mathrm{F}_{2}$ & 2.91 & 1.93 & 1.87 & 1.46 & 1.69 & 1.63 \\
\hline $\mathrm{C}_{2} \mathrm{H}_{2} \ldots \mathrm{ClF}$ & 3.72 & 2.95 & 4.71 & 4.37 & 4.64 & 4.50 \\
\hline$H C N \ldots C l F$ & 5.32 & 4.84 & 5.97 & 5.65 & 5.93 & 5.74 \\
\hline $\mathrm{NH}_{3} \ldots \mathrm{Cl}_{2}$ & 6.00 & 4.87 & 5.51 & 5.06 & 5.31 & 5.20 \\
\hline $\mathrm{H}_{2} \mathrm{O} \ldots \mathrm{ClF}$ & 5.32 & 5.85 & 6.49 & 5.88 & 6.07 & 5.96 \\
\hline $\mathrm{NH}_{3} \ldots \mathrm{ClF}$ & 12.59 & 11.14 & 12.49 & 11.82 & 12.08 & 11.90 \\
\hline MAD & 0.80 & 0.35 & 0.84 & 0.54 & 0.77 & 0.57 \\
\hline
\end{tabular}

\section{2. $D I 6 / 04$.}

(Table 4) shows the evolution of $D_{e}(\mathrm{kcal} / \mathrm{mol})$ for different methods. Here, the MAD of the hybrid LDA/MP2 and PBE/MP2 results $(0.33$ and $0.36 \mathrm{kcal} / \mathrm{mol}$, respectively) are significantly smaller than values obtained with standard LDA $(3.93 \mathrm{kcal} / \mathrm{mol})$ but only moderately smaller than standard PBE $(0.47 \mathrm{kcal} / \mathrm{mol})$. In comparison, the MP2 MAD $(0.24 \mathrm{kcal} / \mathrm{mol})$ is of the same order of magnitude 
as the hybrid ones. In this database as for the former one, both hybrid MADs are very close to each other.

Table 4. Dissociation energies $(\mathrm{kcal} / \mathrm{mol})$ for DI6/04 compounds with different approaches using MG3S basis sets with (cp) and without (no-cp) counterpoise correction.

\begin{tabular}{|c|c|c|c|c|c|c|c|c|c|c|c|}
\hline & \multicolumn{2}{|c|}{ LDA } & \multicolumn{2}{|c|}{$\mathrm{PBE}$} & \multicolumn{2}{|c|}{ LDA/MP2 } & \multicolumn{2}{|c|}{ PBE/MP2 } & \multicolumn{2}{|c|}{ MP2 } & \multirow[t]{2}{*}{ Ref $[6}$. \\
\hline & no-cp & $\mathrm{cp}$ & no-cp & $\mathrm{cp}$ & no-cp & $\mathrm{cp}$ & no-cp & $\mathrm{cp}$ & no-cp & $\mathrm{cp}$ & \\
\hline$\left(\mathrm{H}_{2} \mathrm{~S}\right)_{2}$ & 4.34 & 4.09 & 1.86 & 1.69 & 1.90 & 1.72 & 1.90 & 1.73 & 1.88 & 1.55 & 1.66 \\
\hline$(\mathrm{HCl})_{2}$ & 5.00 & 4.73 & 2.19 & 1.99 & 2.23 & 2.01 & 2.25 & 2.04 & 2.24 & 1.81 & 2.01 \\
\hline $\mathrm{HCl} \cdots \mathrm{H}_{2} \mathrm{~S}$ & 8.14 & 7.81 & 4.45 & 4.19 & 3.85 & 3.57 & 3.86 & 3.59 & 3.75 & 3.20 & 3.35 \\
\hline $\mathrm{CH}_{3} \mathrm{Cl} \cdots \mathrm{HCl}$ & 7.92 & 7.63 & 3.58 & 3.37 & 3.89 & 3.63 & 3.97 & 3.72 & 3.90 & 3.28 & 3.55 \\
\hline$H C N \cdots \mathrm{CH}_{3} \mathrm{SH}$ & 6.91 & 6.73 & 3.71 & 3.60 & 4.07 & 4.07 & 4.10 & 4.10 & 3.91 & 3.44 & 3.59 \\
\hline $\mathrm{CH}_{3} \mathrm{SH} \cdots \mathrm{HCl}$ & 11.27 & 10.93 & 6.15 & 5.87 & 5.61 & 5.29 & 5.64 & 5.32 & 5.46 & 4.69 & 4.16 \\
\hline MAD & 4.21 & 3.93 & 0.60 & 0.47 & 0.54 & 0.33 & 0.57 & 0.36 & 0.47 & 0.24 & \\
\hline
\end{tabular}

In (Table 5), the differences between the geometries of the hybrid calculations and the reference geometries are small compared to corresponding differences with pure functionals. This is another proof of the better accuracy of the hybrid methods compared to standard DFT. We can also see that the MAD is nearly the same between LDA/MP2 and PBE/MP2.

Table 5. Intermolecular distances (A) for DI6/04 compounds with different approaches using MG3S basis sets with (cp) and without (no-cp) counterpoise correction.

\begin{tabular}{|c|c|c|c|c|c|c|c|c|c|c|c|}
\hline & \multicolumn{2}{|c|}{ LDA } & \multicolumn{2}{|c|}{$\mathrm{PBE}$} & \multicolumn{2}{|c|}{ LDA/MP2 } & \multicolumn{2}{|c|}{$\overline{\mathrm{PBE}} / \mathrm{MP} 2$} & \multicolumn{2}{|c|}{ MP2 } & \multirow[t]{2}{*}{ Ref $[34$} \\
\hline & no-cp & $\mathrm{cp}$ & no-cp & $\mathrm{cp}$ & no-cp & $\mathrm{cp}$ & no-cp & $\mathrm{cp}$ & no-cp & $\mathrm{cp}$ & \\
\hline$\left(\mathrm{H}_{2} \mathrm{~S}\right)_{2}$ & 3.752 & 3.756 & 4.054 & 4.083 & 4.106 & 4.136 & 4.096 & 4.096 & 4.110 & 4.184 & 4.115 \\
\hline$(\mathrm{HCl})_{2}^{2}$ & 3.464 & 3.470 & 3.722 & 3.742 & 3.774 & 3.779 & 3.767 & 3.774 & 3.778 & 3.742 & 3.787 \\
\hline $\mathrm{HCl} \cdots \mathrm{H}_{2} \mathrm{~S}$ & 3.438 & 3.448 & 3.612 & 3.622 & 3.699 & 3.708 & 3.694 & 3.708 & 3.731 & 3.779 & 3.756 \\
\hline $\mathrm{CH}_{3} \mathrm{Cl} \cdots \mathrm{HCl}$ & 3.330 & 3.335 & 3.574 & 3.566 & 3.571 & 3.592 & 3.558 & 3.570 & 3.599 & 3.644 & 3.611 \\
\hline $\mathrm{HCN} \mathrm{CCH} \mathrm{CH}_{3} \mathrm{SH}$ & 3.295 & 3.297 & 3.625 & 3.645 & 3.563 & 3.565 & 3.548 & 3.549 & 3.597 & 3.645 & 3.616 \\
\hline $\mathrm{CH}_{3} \mathrm{SH} \cdots \mathrm{HCl}$ & 3.315 & 3.320 & 3.489 & 3.495 & 3.538 & 3.550 & 3.533 & 3.545 & 3.571 & 3.621 & 3.607 \\
\hline MAD & 0.316 & 0.311 & 0.072 & 0.066 & 0.040 & 0.034 & 0.049 & 0.041 & 0.017 & 0.036 & \\
\hline
\end{tabular}

\subsection{WI9/04.}

For these reactions, the MAD values for hybrid methods with counterpoise correction are bigger than without (see Table 6). For example, the PBE/MP2 MAD is 0.10 with $\mathrm{CP}$ and $0.05 \mathrm{kcal} / \mathrm{mol}$ without. This trend is also observed for MP2, and has already been pointed out by Zhao et al [6] . Our hybrid LDA/MP2 (0.10) and PBE/MP2 $(0.10 \mathrm{kcal} / \mathrm{mol})$ values are closer to the reference as compared to LDA and MP2 values (0.67 and 0.14 respectively). Besides, the both LDA/MP2 and PBE/MP2 MADs are the same.

Table 6. Dissociation energies (kcal/mol) for WI9/04 compounds with different approaches using MG3S basis sets with (cp) and without (no-cp) counterpoise correction.

\begin{tabular}{|c|c|c|c|c|c|c|c|c|c|c|c|}
\hline & \multicolumn{2}{|c|}{ LDA } & \multicolumn{2}{|c|}{$\overline{\mathrm{PBE}}$} & \multicolumn{2}{|c|}{ LDA/MP2 } & \multicolumn{2}{|c|}{$\overline{\mathrm{PBE}} / \mathrm{MP} 2$} & \multicolumn{2}{|c|}{ MP2 } & \multirow[t]{2}{*}{ Ref $[6]$} \\
\hline & no-cp & $\mathrm{cp}$ & no-cp & $\mathrm{cp}$ & no-cp & $\mathrm{cp}$ & no-cp & cp & no-cp & $\mathrm{cp}$ & \\
\hline $\mathrm{NeHe}$ & 0.40 & 0.33 & 0.13 & 0.09 & 0.03 & 0.01 & 0.02 & 0.01 & 0.05 & 0.01 & 0.04 \\
\hline $\mathrm{ArHe}$ & 0.39 & 0.35 & 0.10 & 0.07 & 0.06 & 0.04 & 0.05 & 0.04 & 0.05 & 0.03 & 0.06 \\
\hline $\mathrm{Ne}_{2}$ & 0.59 & 0.42 & 0.21 & 0.11 & 0.09 & 0.01 & 0.08 & 0.01 & 0.18 & 0.01 & 0.08 \\
\hline$A r N e$ & 0.71 & 0.56 & 0.23 & 0.14 & 0.13 & 0.04 & 0.12 & 0.04 & 0.21 & 0.03 & 0.13 \\
\hline $\mathrm{CH}_{4} \cdots \mathrm{Ne}$ & 0.84 & 0.64 & 0.27 & 0.15 & 0.18 & 0.06 & 0.17 & 0.06 & 0.29 & 0.04 & 0.22 \\
\hline $\mathrm{C}_{6} \mathrm{H}_{6} \cdots \mathrm{Ne}$ & 1.53 & 1.13 & 0.39 & 0.19 & 0.51 & 0.21 & 0.50 & 0.22 & 0.63 & 0.13 & 0.47 \\
\hline$\left(\mathrm{CH}_{4}\right)_{2}$ & 1.23 & 1.14 & 0.16 & 0.16 & 0.41 & 0.36 & 0.44 & 0.39 & 0.41 & 0.32 & 0.51 \\
\hline$\left(\mathrm{C}_{2} \mathrm{H}_{2}\right)_{2}$ & 2.54 & 2.71 & 1.02 & 1.23 & 1.48 & 1.37 & 1.53 & 1.41 & 1.49 & 1.27 & 1.34 \\
\hline$\left(\mathrm{C}_{2} \mathrm{H}_{4}\right)_{2}$ & 3.10 & 3.03 & 0.56 & 0.53 & 1.52 & 1.32 & 1.60 & 1.51 & 1.57 & 1.17 & 1.42 \\
\hline MAD & 0.78 & 0.67 & 0.22 & 0.20 & 0.05 & 0.10 & 0.06 & 0.10 & 0.09 & 0.14 & \\
\hline
\end{tabular}

(Table 7) shows the geometries for different methods with counterpoise correction. The hybrid LDA/MP2 and PBE/MP2 MADs are the smallest ones, and are basically the same $(0.18$ and $0.17 \mathrm{kcal} / \mathrm{mol}$ respectively $)$. 
Table 7. Intermolecular distances (A) for WI9/04 compounds with different approaches using MG3S basis sets with counterpoise correction.

\begin{tabular}{lcccccc}
\hline & LDA & PBE & LDA/MP2 & PBE/MP2 & MP2 & Ref $[34]$ \\
\hline $\mathrm{NeHe}$ & 2.476 & 2.911 & 3.449 & 3.433 & 3.059 & 3.031 \\
$\mathrm{ArHe}$ & 2.937 & 3.495 & 3.617 & 3.604 & 3.708 & 3.480 \\
$\mathrm{Ne}_{2}$ & 2.583 & 3.156 & 3.553 & 3.544 & 3.648 & 3.091 \\
$\mathrm{ArNe}$ & 3.016 & 3.490 & 3.494 & 3.493 & 3.868 & 3.489 \\
$\mathrm{CH}_{4} \cdots N_{e}$ & 2.971 & 3.536 & 3.683 & 3.723 & 3.769 & 3.486 \\
$\mathrm{C}_{6} \mathrm{H}_{6} \cdots N_{e}$ & 3.183 & 3.784 & 3.692 & 3.660 & 3.950 & 3.509 \\
$\left(\mathrm{CH}_{4}\right)_{2}$ & 3.329 & 4.040 & 3.759 & 3.712 & 3.788 & 3.615 \\
$\left(\mathrm{C}_{2} \mathrm{H}_{2}\right)_{2}$ & 3.530 & 3.867 & 3.442 & 3.453 & 3.477 & 3.456 \\
$\left(\mathrm{C}_{2} \mathrm{H}_{4}\right)_{2}$ & 3.551 & 4.154 & 3.849 & 3.856 & 3.892 & 3.834 \\
$\mathrm{MAD}$ & & & & & & \\
\hline
\end{tabular}

\section{4. $\mathrm{HB6} / 04$.}

For this last subset, the hybrid no-cp MADs are not as good as for the previous ones (see Table 8). Indeed, in this case the MP2 MAD is $0.27 \mathrm{kcal} / \mathrm{mol}$, while it increases up to 1.55 for LDA/MP2 and 1.37 for PBE/MP2. Using the counterpoise correction leads to better results. With this correction, the LDA/MP2 MAD is reduced to $0.94 \mathrm{kcal} / \mathrm{mol}$ (compared to 1.55 ), the $\mathrm{PBE} / \mathrm{MP} 2 \mathrm{MAD}$ to $0.81 \mathrm{kcal} / \mathrm{mol}$ (compared to 1.37). These results are close to the counterpoise corrected MP2 MAD $(0.91 \mathrm{kcal} / \mathrm{mol})$. However, the PBE MAD $(0.29 \mathrm{kcal} / \mathrm{mol})$ is better than the hybrid ones. The difference between the LDA/MP2 MAD and the PBE/MP2 MAD is very small again. The MADs for hybrid methods are less sensitive to the basis set quality than for standard MP2 and DFT methods. For example, the differences of the MADs between the most accurate basis set (aug-cc-pVTZ) and the least accurate one (DIDZ) are 0.64 and $1.95(\mathrm{kcal} / \mathrm{mol})$ for the MP2 and LDA calculations, respectively, and reduces to $0.06(\mathrm{kcal} / \mathrm{mol})$ for LDA/MP2. We also note that the difference of $D_{e}$ with/without geometry optimization is weak: in our case, we obtained a MAD of 0.87 for optimized PBE/MP2 calculations compared to $0.97(\mathrm{kcal} / \mathrm{mol})$ without optimisation [4].

Table 8. Dissociation energies (kcal/mol) for HB6/04 compounds with different approaches using MG3S basis sets with (cp) and without (no-cp) counterpoise correction.

\begin{tabular}{|c|c|c|c|c|c|c|c|c|c|c|c|}
\hline & \multicolumn{2}{|c|}{ LDA } & \multicolumn{2}{|c|}{$\overline{\mathrm{PBE}}$} & \multicolumn{2}{|c|}{ LDA/MP2 } & \multicolumn{2}{|c|}{$\overline{\mathrm{PBE} / \mathrm{MP} 2}$} & \multicolumn{2}{|c|}{ MP2 } & \multirow[t]{2}{*}{ Ref [6] } \\
\hline & no-cp & $\mathrm{cp}$ & $\mathrm{co}-\mathrm{cp}$ & $\mathrm{cp}$ & no-cp & $\mathrm{cp}$ & no-cp & $\mathrm{cp}$ & no-cp & $\mathrm{cp}$ & \\
\hline$\left(\mathrm{NH}_{3}\right)_{2}$ & 6.13 & 5.93 & 3.34 & 3.16 & 3.50 & 3.25 & 3.51 & 3.27 & 3.33 & 2.91 & 3.15 \\
\hline$(H F)_{2}$ & 8.59 & 8.01 & 5.11 & 4.67 & 5.49 & 4.93 & 5.31 & 4.85 & 4.91 & 3.96 & 4.57 \\
\hline$\left(\mathrm{H}_{2} \mathrm{O}\right)_{2}$ & 9.29 & 8.62 & 5.58 & 5.05 & 6.04 & 5.47 & 5.92 & 5.40 & 5.47 & 4.54 & 4.97 \\
\hline $\mathrm{NH}_{3} \ldots \mathrm{H}_{2} \mathrm{O}$ & 11.42 & 10.99 & 7.25 & 6.89 & 7.40 & 6.96 & 7.30 & 6.90 & 6.78 & 5.97 & 6.41 \\
\hline$\left(\mathrm{HCONH}_{2}\right)_{2}$ & 23.27 & 22.72 & 14.37 & 13.99 & 16.83 & 16.05 & 16.66 & 15.95 & 14.88 & 13.25 & 14.94 \\
\hline$(\mathrm{HCOOH})_{2}$ & 29.29 & 28.19 & 16.94 & 16.24 & 20.33 & 19.19 & 19.70 & 18.67 & 16.29 & 14.10 & 16.15 \\
\hline MAD & 6.30 & 5.71 & 0.59 & 0.29 & 1.55 & 0.94 & 1.37 & 0.81 & 0.27 & 0.91 & \\
\hline
\end{tabular}

(Table 9) shows the counterpoise-corrected optimized geometries for different methods. In this case, the MADs are small for every methods, but the MP2 and PBE MADs are the smallest ones. The LDA/MP2 and PBE/MP2 MADs are the same $(0.06 \mathrm{kcal} / \mathrm{mol})$.

Table 9. Intermolecular distances (A) forHB6/04 compounds with different approaches using MG3S basis sets with counterpoise correction.

\begin{tabular}{lcccccc}
\hline & LDA & PBE & LDA/MP2 & PBE/MP2 & MP2 & Ref [34] \\
\hline$\left(\mathrm{NH}_{3}\right)_{2}$ & 3.272 & 3.257 & 3.230 & 3.221 & 3.247 & 3.267 \\
$(\mathrm{HF})_{2}$ & 2.569 & 2.750 & 2.720 & 2.720 & 2.820 & 2.783 \\
$\left(\mathrm{H}_{2} \mathrm{O}\right)_{2}$ & 2.732 & 2.917 & 2.883 & 2.883 & 2.980 & 2.937 \\
$\mathrm{NH}_{3} \mathrm{HH}_{2} \mathrm{O}$ & 2.757 & 2.910 & 2.902 & 2.901 & 2.974 & 2.967 \\
$(\mathrm{HCONH})_{2}$ & 2.697 & 2.862 & 2.833 & 2.833 & 2.911 & 2.881 \\
$(\mathrm{HCOOH})_{2}$ & 2.478 & 2.633 & 2.600 & 2.614 & 2.720 & 2.702 \\
& & & & & & \\
$\mathrm{MAD}$ & 0.173 & 0.035 & 0.061 & 0.061 & 0.026 & \\
\hline
\end{tabular}




\section{Conclusion}

Analytical gradients for mixed short-range density-functional ( $s r$-DFT) / longrange Hartree-Fock ( $l r$-HF) and $s r$-DFT / long-range Møller-Plesset second-order perturbation theory (lr-MP2) methods have been implemented into the Molpro package of ab-initio programs. This exclusive new feature has been validated on the Truhlar benchmark set of weakly interacting molecules. It is shown that it is now possible to optimize geometries of at least similar quality than with conventional MP2 for cases where standard functionals usually fail. Due to the flexibility of the implementation, large molecular systems are accessible using local correlation methods and gradient corrected short-range functionals. In addition, smaller basis sets than for conventional MP2 are sufficient due to the weak basis-set dependence of the mixed method. However, there is still room for improvement with respect to the efficiency and the scaling of the code. Work towards this direction is currently in progress.

\section{Acknowlegdments}

S.C. thanks the Deutsch-Französische Hochschule (DFH-UFA) and the Ecole Doctorale des Sciences de la Matière of the University Toulouse 3 for financial support. T.L. thanks the Alexander von Humboldt Foundation for visiting grants to the Institut für Theoretische Chemie, University of Stuttgart.

\section{References}

[1]P. Pulay, Mol. Phys. 17, 197 (1969).

[2]T. Helgaker and P.R. Taylor, in Modern Electronic Structure Theory, edited by D. Yarkony (World Scientific, Singapore, 1995).

[3]T. Leininger, H. Stoll, H.J. Werner and A. Savin, Chem. Phys. Lett. 275, 151 (1997).

[4]E. Goll, H.J. Werner and H. Stoll, Chem. Phys. 346, 257 (2008).

[5]E. Goll, H.J. Werner and H. Stoll, Phys. Chem. Chem. Phys. 7, 3917 (2005).

[6]Y. Zhao and D.G. Truhlar, J. Chem. Theory Comput. 1, 415 (2005).

[7] M. Levy, Proc. Acad. Sci. U.S.A. 76, 6062 (1979).

[8]H.-J. Werner, P. J. Knowles, R. Lindh, F. R. Manby, M. Schütz and others, MOLPRO, version 2009.1, a package of ab initio programs 2009.

[9] A. El Azhary, G. Rauhut, P. Pulay and H.J. Werner, J. Chem. Phys. 108, 5185 (1998).

[10]M. Schütz, H.J. Werner, R. Lindh and F.R. Manby, J. Chem. Phys. 121, 737 (2004).

[11] M.J. Frisch, M. Head-Gordon and J.A. Pople, Chem. Phys. Lett. 166, 275 (1990).

[12] J. Gerratt and I.M. Mills, J. Chem. Phys. 49, 1719 (1968).

[13]Y. Osamura, Y. Yamaguchi, P. Saxe, D.J. Fox, M.A. Vincent and H.F. Schaefer, J. Mol. Struct. 103, $183(1983)$.

[14]R. Lindh, Theor. Chim. Acta. 85, 423 (1992).

[15]R. Lindh, U. Ryu and B. Liu, J. Chem. Phys. 95, 5889 (1991).

[16] J.P. Perdew and Y. Wang, Phys. Rev. B 45, 13244 (1992).

17]P. Gori-Giorgi and J.P. Perdew, Phys. Rev. B 64, 155102 (2001).

[18] L. Zecca, P. Gori-Giorgi, S. Moroni and G.B. Bachelet, Phys. Rev. B 70, 205127 (2004).

[19] S. Paziani, S. Moroni, P. Gori-Giorgi and G.B. Bachelet, Phys. Rev. B 73, 155111 (2006).

[20] A. Savin, in Recent developments and applications of modern Density Functional Theory, edited by J. M. Seminario (Elsevier, Amsterdam, 1996).

[21]E. Goll, H.J. Werner and H. Stoll, Phys. Chem. Chem. Phys. 7, 3917 (2005).

[22] V.A. Rassolov, J.A. Pople, M.A. Ratner, P.C. Redfern and L.A. Curtiss, J. Comp Chem. 22, 976 (2001).

[23] W.J. Hehre, L. Radom, P.v.R. Schleyer and J.A. Pople, Ab Initio Molecular Orbital Theory (Wiley, New York, 1986).

[24]T. Clark, J. Chandrasekhar, G.W. Spitznagel and P.v.R. Schleyer, J. Comp. Chem. 4, 294 (1983).

[25] R. Krishnan, J.S. Binkley, R. Seeger and J.A. Pople, J. Chem. Phys. 72, 650 (1980).

[26] M.J. Frisch, J.A. Pople and J.S. Binkley, J. Chem. Phys. 80, 3265 (1984).

[27]P.L. Fast, M.L. Sanchez and D.G. Truhlar, Chem. Phys. Lett. 306, 407 (1999).

[28]B.J. Lynch, Y. Zhao and D.G. Truhlar, J. Phys. Chem. A. 107, 1384 (2003).

[29] L.A. Curtiss, C. Redfern, K. Raghavachari, V.A. Rassolov and J.A. Pople, J. Chem. Phys. 110, 4703 (1999).

[30]T.H. Dunning Jr., J. Chem. Phys. 90, 1007 (1989). 
[31]R.A. Kendall, T.H. Dunning Jr and R.J. Harrison, J. Chem. Phys. 96, 6796 (1992).

[32] D.E. Woon, J. Chem. Phys. 100, 2838 (1994).

[33] A. Boese and J.M.L. Martin, J. Chem. Phys. 121, 3405 (2004).

[34]D.G. Truhlar http://comp. chem.umn.edu/truhlar/database_noncov/noncovalent.htm.

1

2

3

4

5

6

7

8

9

10

11

12

13

14

15

16

17

18

19

20

21

22

23

24

25

26

27

28

29

30

31

32

33

34

35

36

37

38

39

40

41

42

43

44

45

46

47

48

49

50

51

52

53

54

55

56

57

58

59

60 
Prof. Thierry Leininger

Toulouse, June $27^{\text {st }} 2010$

Laboratoire de Chimie et Physique Quantiques

IRSAMC

Université Paul Sabatier - Toulouse 3

118, route de Narbonne

31062 Toulouse Cedex

+33(0)561556152

Thierry.Leininger@irsamc.ups-tlse.fr

Ref.: Manuscript nºTMPH-2010-0224

Dear Editor,

since the submission of the above manuscript, we found a cut/paste error in one of the table. Actually, in Table 4 (Dissociation enegies for the DI6/04 set), the "LDA/MP2 cp" column was wrong: the values appearing in the submitted paper were these from column "MP2/cp" of Table 1. As you will see this doesn't change the conclusions of the present work as this erroneous substitution must have appeared close to the end of the manuscript preparation.

I send enclosed a corrected version of the manuscript.

I am very sorry for this.

Please send all my apologies to the referees.

With my best regards,

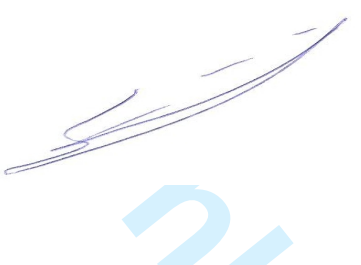

Thierry Leininger 